\title{
Exploring novel features and decision rules to identify cardiovascular autonomic neuropathy using a Hybrid of Wrapper-Filter based feature selection
}

\author{
Shamsul Huda ${ }^{\# 1}$, Herbert Jelinek ${ }^{* 2}$, Biplob Ray ${ }^{\# 3}$, Andrew Stranieri ${ }^{\# 4}$, John Yearwood ${ }^{\# 5}$ \\ \# CIAO, GSITMS, University of Ballarat, Victoria, Australia \\ ${ }^{1}$ s.huda, ${ }^{4}$ a.stranieri, ${ }^{5}$ j.yearwood $\}$ aballarat.edu.au \\ ${ }^{*}$ School of Community Health, Centre for Research in Complex Systems, Charles Sturt University \\ ${ }^{2}$ HJelinek@csu.edu.au
}

\begin{abstract}
Cardiovascular autonomic neuropathy (CAN) is one of the important causes of mortality among diabetes patients. Statistics shows that more than $22 \%$ of people with type 2 diabetes mellitus suffer from CAN and which in turn leads to cardiovascular disease (heart attack, stroke). Therefore early detection of CAN could reduce the mortality. Traditional method for detection of CAN uses Ewing's algorithm where five noninvasive cardiovascular tests are used. Often for clinician, it is difficult to collect data from for the Ewing Battery patients due to onerous test conditions. In this paper, we propose a hybrid of wrapper-filter approach to find novel features from patients' ECG records and then generate decision rules for the new features for easier detection of CAN. In the proposed feature selection, a hybrid of filter (Maximum Relevance, MR) and wrapper (Artificial Neural Net Input Gain Measurement Approximation ANNIGMA) approaches (MR-ANNIGMA) would be used. The combined heuristics in the hybrid MRANNIGMA takes the advantages of the complementary properties of the both filter and wrapper heuristics and can find significant features. The selected features set are used to generate a new set of rules for detection of CAN. Experiments on real patient records shows that proposed method finds a smaller set of features for detection of CAN than traditional method which are clinically significant and could lead to an easier way to diagnose CAN.
\end{abstract}

\section{INTRODUCTION}

Cardiovascular autonomic neuropathy (CAN) occurs due to damage in the autonomic nerve fibres to the heart and is one of the important causes of mortality among diabetes patients $[1,2]$. Statistics shows that more than $22 \%$ of people with type 2 diabetes mellitus (DM) suffer from CAN which in turn leads to impaired regulation of blood pressure and heart rate variability (HRV) and $75 \%$ of diabetic-people die from cardiovascular disease [3]. A survey on a group of population of different age, sex, and varying duration with diabetes shows that the mortality of diabetic patients with CAN increased steadily over the 8 -year period $(6 \%$ after 2 years, $14 \%$ after 4 years, $17 \%$ after 6 years, and $23 \%$ after 8 years) [4]. Therefore early detection of CAN could reduce mortality and enhance health. The traditional method for detection of CAN uses Ewing's algorithm [6, 7] where five simple non invasive cardiovascular tests/ features such as (Valsalva manoeuvre, heart rate response to deep breathing and standing up, blood pressure response to standing up and sustained handgrip) are used. The panel on consensus meetings on Diabetic Neuropathy, San Antonio-1988 [6, 7] also recommended five tests for detection of CAN (heart rate response to 1) deep breathing, 2) standing, and 3) the Valsalva manoeuvre and two tests of blood pressure control were also recommended: blood pressure response to 1) standing or passive tilting and 2) sustained handgrip. Often it is difficult for clinicians to collect data for the Ewing Battery from patients due to onerous test conditions.

In this paper, we would employ novel data mining techniques such as feature selection and rule generation to discover a new set of features and generate decision rules for the detection of CAN using a combination of patients ECG records and traditional features. We propose a hybrid of wrapper-filter approach for extracting novel features from the morphological data of patients ECG records and then generate decision rules from new features for detection of CAN using decision tree. Our earlier research [5] shows that a hybrid of wrapper-filter heuristic significantly improves the performances over the independent wrapper and filter approaches in feature selection applications [5]. In this paper we use this concept of wrapper-filter hybrid heuristic [5] for pre-filtering of redundant features in the diagnosis of CAN. Our proposed approach uses a hybrid of filter (Maximum Relevance, MR) and wrapper (Artificial Neural Net Input Gain Measurement Approximation ANNIGMA) approaches (MR-ANNIGMA). The combined heuristics in the hybrid MR-ANNIGMA takes the advantages of the complementary properties of the both filter and wrapper heuristics and can find most significant features which later can be used to generate new set of simplified rules for detection of CAN. The rules are generated by standard decision trees.

The rest of the paper is organized as follows. The next section introduces some related literature. Section III describes the data set used in this paper and some background terminology related to ECG feature extraction. The proposed hybrid of wrapper-filter feature selection algorithm and decision rule generation process in Section IV. Section V presents experimental results and discussion. Conclusions of this study are presented in the last section. 


\section{RELATED WORK}

Ewing et al. [6, 7] proposed five simple autonomic function tests. Many others $[9,10]$ also used these five tests for CAN. The tests are: HR response to Valsalva manoeuvre (sitting, VA_HR), HR variation during deep breathing (DB_HR), BP response to sustained handgrip (Sitting), immediate HR response to standing (Lying to standing), and BP response to standing (Lying to standing). Ewing [6, 7] mentions three different ranges for these tests such as normal, borderline and abnormal. CAN is then determined depending on the number of tests either borderline or abnormal into mild, moderate, severe and atypical. The results are used to categorise patients into different types of CAN $[6,7]$.

\section{DATA SET AND ECG FEATURES}

The data was collected from people attending the diabetes complications screening research initiative (DiScRi) at Charles Sturt University. Ethics was approved by the universities ethics in human research committee prior to commencing with the project. Participants were recruited through media advertisement and were requested to fast from midnight the previous day as well as abstain from smoking, coffee and alcohol for 24 hours. Measures were undertaken between 9:00 am and 12 noon. There were no exclusion criteria. All participants had their age, sex and diabetes status recorded as well as blood pressure (BP), body-mass index (BMI), blood glucose level (BGL), and cholesterol profile. 12lead ECG was recorded for $10 \mathrm{sec}$. All participants undertook the Ewing battery $[6,7]$ consisting of 5 tests. From the 12-lead ECG we measured QRS, PQ, QTc and QTd intervals as well as heart rate and QRS axis. The five Ewing tests used were changes in heart rate associated with lying to standing, deep breathing and valsalva manoeuvre and changes in blood pressure associated with hand grip and lying to standing. Data of 291 participants attending DiScRi were selected on the basis that they had a complete Ewing battery result (5 tests). Demographic details are listed in Table-I. Eleven people reported having had a heart attack, 14 reported atrial fibrillation and 38 had palpitations.

TABLE I: DEMOGRAPHIC DATA FOR PARTICIPANTS

\begin{tabular}{|l|l|}
\hline Variable & Result \\
\hline Number screened & 291 \\
\hline Sex (f/m) & $182 / 109$ \\
\hline Age \pm sd (yrs) & $64.1 \pm 11$ \\
\hline Diabetes & 67 \\
\hline Smoking & 4 \\
\hline Alcohol & 24 \\
\hline SBP* \pm sd $(\mathrm{mmHg})$ & $129.4 \pm 18$ \\
\hline DSP $\pm \mathrm{sd}(\mathrm{mmHg})$ & $76.2 \pm 11$ \\
\hline BGL $\pm \mathrm{sd}(\mathrm{mmol} / \mathrm{L})$ & $5.72 \pm 2.1$ \\
\hline
\end{tabular}

ECG features are used in combination with the traditional Ewing features. ECG features are extracted from the 10 seconds recording. The following ECG features were used (Fig. 1.):

QRS Complex $=$ QRS complex represents the ventricular activity of the heart (the depolarization of the ventricles) and its duration is known as the QRS duration. PQ Interval = PQ interval is the time elapsed between the beginning of the $\mathrm{P}$ wave at the beginning of the next QRS complex.

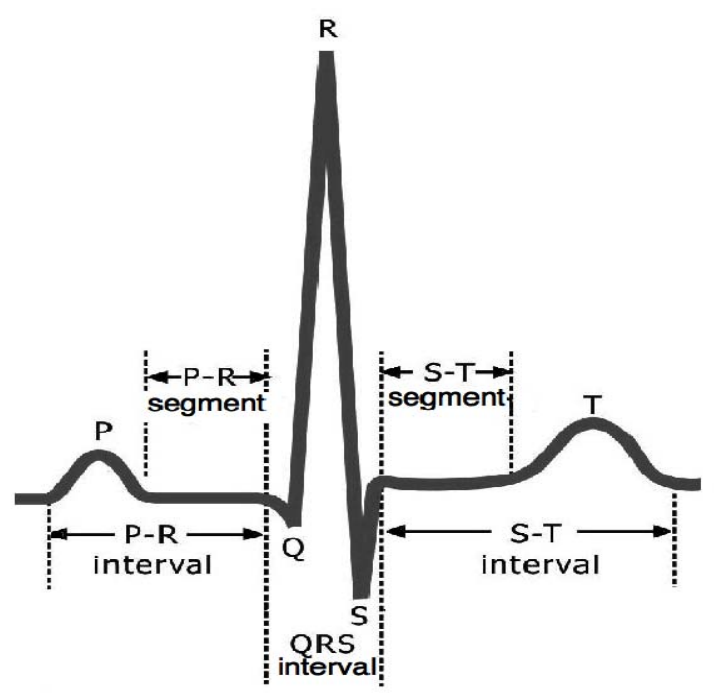

Fig. 1. Features from ECG signals used in CAN detection

QTc $=$ QT interval is the longest distance between the Q wave and the T wave. QTc is the corrected QT interval which is the period from the beginning of the QRS complex to the end of the $\mathrm{T}$ wave. It reflects the refractory period of the heart. QTc is QT interval corrected for heart rate.

QTc $>1 / 2=$ is the cut-off for abnormal beat

QTd = QT dispersion (maximum QT interval minus minimum QT interval) is an approximate measure of a general abnormality of repolarisation.

QRS axis degree is the deflection of the electrical axis of the heart in degrees to the right or left

Grade $=$ grading for referral requirement.

\section{IV.PROPOSED HYBRID OF WRAPPER-FILTER BASED FEATURE SELECTION AND DECISION RULES GENERATION TO IDENTIFY CARDIOVASCULAR AUTONOMIC NEUROPATHY}

In the proposed approach, we would investigate new features including patients ECG records which have not been explored in the literature. Then we extract new rules for detection of Cardiovascular Autonomic Neuropathy (CAN) using the selected features and corresponding records. The proposed methodology can be described by Fig 2 .

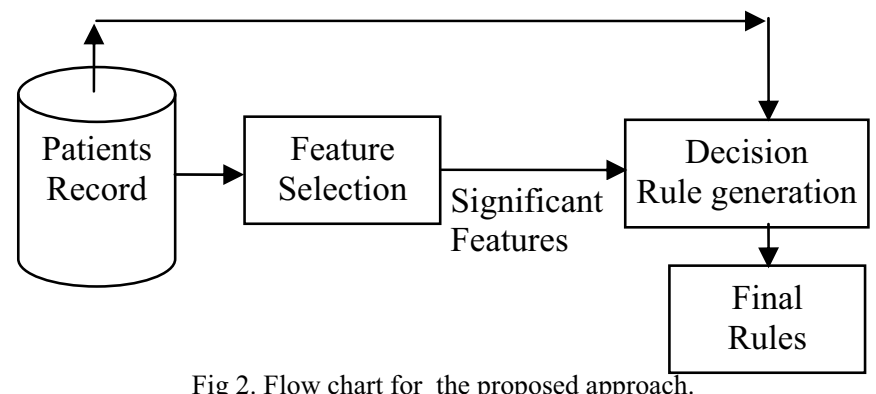

In the next section, the detailed methodology is described. 


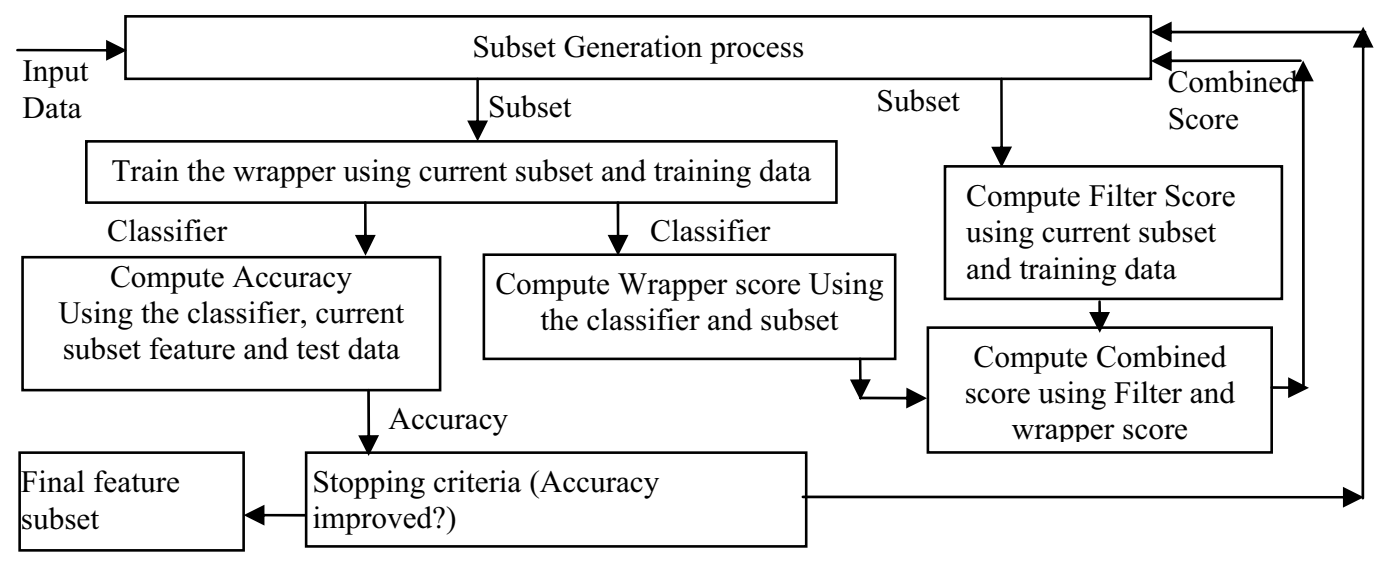

Fig 3. Flow chart for hybrid of wrapper and Filter feature selection in the proposed approaches

\section{A. First step: Feature selection:}

Standard filter approaches do not guarantee $[4,11,12]$ that selected feature subset will do better in classification/prediction tasks. In contrast, the wrapper approach [4], [11], [12] [13], [14] uses a predetermined induction algorithm and repeated execution of the induction algorithm in the search process incurs a high computational cost in the wrapper approach. Our earlier research shows that hybrid of wrapper-filter approaches [5] can introduce the filter heuristic in the wrapper stage and can take advantages of both approaches. This technique [5] can direct the search process to the more promising space in the wrapper and find more significant features subset. Therefore, in the proposed approach we have combined mutual information based Maximum Relevance (MR) filter heuristics and Artificial Neural Network Input Gain Measurement Approximation (ANNIGMA) based wrapper heuristic in the wrapper stage. The feature selection process can be described by fig 3 and Algorithm-1.

\section{B. Artificial Neural Network Input Gain Measurement Approximation (ANNIGMA)}

Artificial Neural Network Input Gain Measurement Approximation (ANNIGMA)[12] is a weight analysis based wrapper heuristic that ranks features by relevance based on the weight associated with feature in a Neural Network based wrapper approach. Features that are irrelevant or redundant will produce more error than relevant features. Therefore, during training, weights of noisy features are controlled in such a way that they contribute to the output as least as possible. ANNIGMA [12] is based on the above strategy of the training algorithm. For a two layer Multi Layer Perceptron (MLP) Neural Network, if i, j, $\mathrm{k}$ are the input, hidden and output layer and Q is a logistic activation function (1) of the first layer and second layer has a linear function, then output of the network is as (2).

$$
\begin{aligned}
& Q(x)=(1 /(1+\exp (-x))) \\
& O_{k}=\sum_{j} Q\left(\sum_{i} A_{i} \times W_{i j}\right) \times W_{j k}
\end{aligned}
$$

Then local gain is defined as (3)

$$
L G_{i k}=\frac{\Delta O_{k}}{\Delta A_{i}}
$$

According to C.N. Hsu and H.J. Huang et.al. [12], the local gain can be written in terms of network weight as (4):

$$
L G_{i k}=\sum_{j}\left|W_{i j} \times W_{j k}\right|
$$

Then ANNINGMA score for feature-i $\left(F_{i}\right)$ is the local gain (LG) normalized based on a unity scale as (5)

$$
\operatorname{ANNIGMA}\left(F_{i}\right)=\frac{L G_{i k}}{\max \operatorname{imum}_{(i)} L G_{i k}}
$$

\section{Maximum Relevance (MR) filter heuristics}

Relevant features provide more information about the class variable than irrelevant features. Therefore mutual information based maximum relevance [8] is a good heuristic to select salient features in data mining area. If $\mathrm{S}$ is a set of features $F_{i}$ and class variable is $c$, the maximum relevance [8] can be defined as (6).

$\max$ imum $\operatorname{Re}$ levance $(S, c)=\frac{1}{|S|} \sum_{f_{i} \in S} I\left(F_{i} ; c\right)$

$I\left(F_{i} ; c\right)$ is the mutual information between $F_{i}$ and class c.

\section{Algorithm-1: Procedure (Hybrid Wrapper-Filter approach for feature selection)}

Input: $D\left(F_{1}, F_{2}, \ldots F_{m}\right) / /$ Training data with $\mathrm{m}$ features

Output: $S_{B E S T}$ //an optimal subset of features

Begin

1. Let $\mathrm{S}=$ whole set of $\mathrm{m}$ features $F_{1}, F_{2}, \ldots F_{m}$

2. $S_{0}=$ Initial set of feature which records all generated subsets with accuracy

// Apply a BE search strategy

3. for $\mathrm{N}=1$ to $\mathrm{m}-1$

4. Current set of feature $S_{\text {current }}=\mathrm{S}$

5. Compute Filter score by (6)

6. for fold $=1$ to $n$

7. Train the network with $S_{\text {current }}$ 
8.

9.

10.

Compute ANNGMA of all features

10. endfor

11. Compute average accuracy of all folds for $S_{\text {current }}$

12. Compute average ANNIGMA of $S_{\text {current }}$ by (7)

13. Compute combined score for every feature in $S_{\text {current }}$ by ( 8 to 9 ) for hybrids

14. Rank the features in $S_{\text {current }}$ using the combined score in descending order

15. $S_{0}=S_{0} \cup S_{\text {curren }}$

16. Update the current feature set $S_{\text {current }}$ by removing the feature with lowest score

17. endfor

18. $S_{B E S T}=$ Find the subset form $S_{0}$ with the highest accuracy.

19. return $S_{B E S T}$

End

D. Computation of combined score in the hybrid wrapperFilter feature selection algorithm: Hybrid of Maximum Relevance and ANNIGMA (MR-ANNIGMA) for the proposed approach

The proposed MR-ANNIGMA uses Artificial Neural Network as the classification algorithm in the wrapper stage. An n-fold cross-validation approach has been used in MRANNIGMA to train the wrapper. In each fold we compute the ANNIGMA score for every feature. Then after training of all folds, the ANNIGMA score is averaged as (7): $\operatorname{ANNIGMA}\left(F_{i}\right)_{\text {average }}=$

$\left(\frac{1}{n}\right)\left(A N N I G M A\left(F_{i}\right)_{1}+. .+\operatorname{ANNIGMA}\left(F_{i}\right)_{n}\right)$

While computing the combined score in the proposed ANNIGMA, the relevance of a feature in the current subset is computed from the individual score which is scaled to the maximum individual relevance of the subset. Thus relevance of a feature in a subset in the hybrid approach is as (8)

$\operatorname{Re}$ levance $\left(F_{i}\right)=\frac{I\left(F_{i} ; c\right)}{\operatorname{maximum}\left(f_{i} \in S\right) I\left(F_{i} ; c\right)}$

The combined score of filter's heuristic and wrapper's heuristic in the proposed MR-ANNIGMA is computed as (9).

Combined Score $(M R-A N N I G M A)=$

$\operatorname{Re}$ levance $\left(F_{i}\right)+\operatorname{ANNIGMA}\left(F_{i}\right)_{\text {average }}$

\section{E. Search strategies and subset generation in the hybrid approach(MR-ANNIGMA)}

The hybrid approach uses a Backward Elimination (BE) search strategy to generate a subset of features. Initially hybrid starts with the full feature set. Subset generation in BE is guided by the wrapper-filter hybrid heuristic score as (9). The combined score computation follows the steps of sub-sections (B-D). In the wrapper stage, an $\mathrm{n}$-fold cross validation approach has been applied in the training of the MLP network. The evaluation criterion of feature subset is based on the average prediction accuracy over n-fold of the wrapper (MLP network). In Algorithm-1, steps-1 to 11 computes the average accuracy over n-folds for the current subset of features. Step12 to step-14 computes the hybrid scores and ranks the features based on their combined score. Step-15 to step-16 generates new subset based on the feature ranking and keep records of evaluated feature subsets with their accuracy. The BE continues until a single feature is remaining in the current subset. The subset with highest accuracies or close to the highest accuracies with fewer features than it is chosen as the final feature subset.

\section{F. Decision Rule Generation for identifying CAN}

Selected features by using the proposed feature selection algorithm along with corresponding patients' records are used to feed to a Standard Decision Tree classifier (SDTs) $[15,16]$. A SDT is a rooted tree with a node called the root that has no incoming edge. Nodes with both incoming and outgoing edges are called internal nodes. Nodes with no outgoing edges are called leaves. The rules are generated from the root of the constructed SDT to one of its leaves and are transformed to a decision rule by co-joining the intermediate nodes' testconditions that forms the antecedent part of the rule and class value of the leaf forms the consequent part of the rule.

\section{EXPERIMENTAL RESULTS AND DISCUSSION}

The proposed approach has been tested on real data set described in the section III. The data is normalized in the range $[-1,1]$. A single hidden layer neural network with 6 hidden neuron, hidden transfer function (tansig) and output transfer function (Purelinear) with a maximum epoc of 300 has been considered as the wrapper.

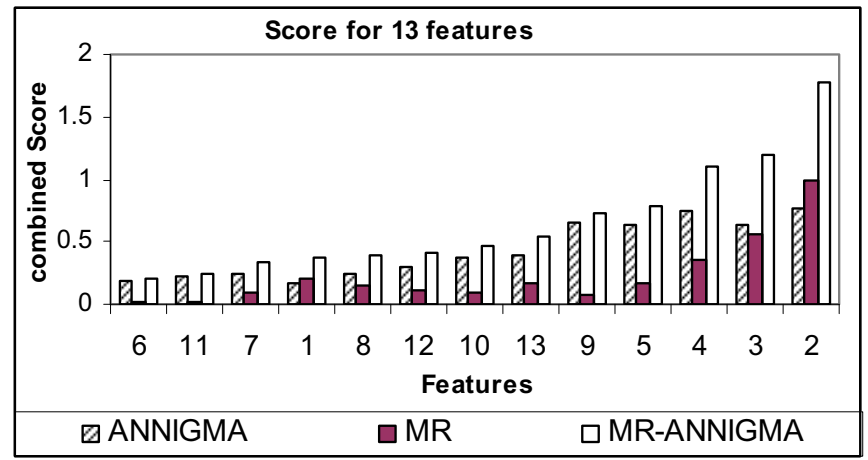

Fig 4. The score graph for BE iteration when total attributes is 13 .

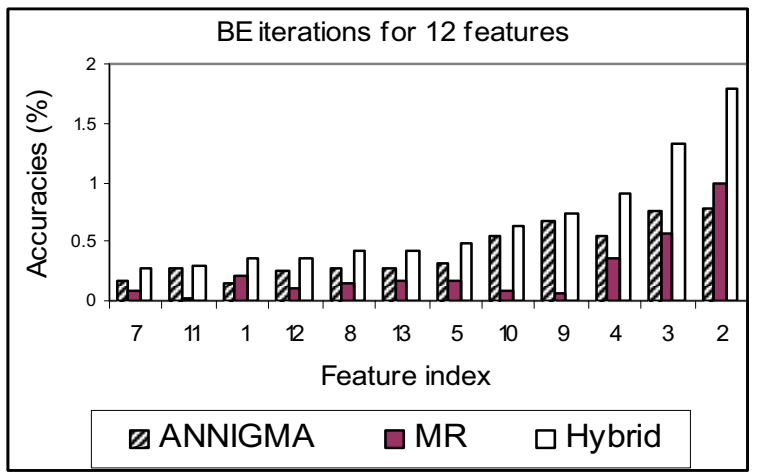

Fig. 5. The score graph for BE iteration when total attributes is 12 . 
TABLE II: ACCURACIES FOR RULE GENERATION PROCESS FOR PROPOSED APPROACH, EWING'S METHOD AND ALL FEATURES

\begin{tabular}{|l|l|l|l|l|l|l|l|l|l|}
\hline Trials & \multicolumn{3}{|l|}{ Proposed Approach } & \multicolumn{3}{l|}{ Only Ewing Features } & \multicolumn{2}{|l|}{ All Features } \\
\hline & $\begin{array}{l}\text { Training } \\
\text { Error } \\
\text { rate (\%) }\end{array}$ & $\begin{array}{l}\text { Test } \\
\text { Error } \\
\text { rate (\%) }\end{array}$ & $\begin{array}{l}\text { Tree } \\
\text { Size }\end{array}$ & $\begin{array}{l}\text { Training } \\
\text { Error } \\
\text { rate (\%) }\end{array}$ & $\begin{array}{l}\text { Test } \\
\text { Error } \\
\text { rate } \\
\mathbf{( \% )}\end{array}$ & $\begin{array}{l}\text { Tree } \\
\text { Size }\end{array}$ & $\begin{array}{l}\text { Training } \\
\text { Error } \\
\text { rate (\%) }\end{array}$ & $\begin{array}{l}\text { Test } \\
\text { Error } \\
\text { rate } \\
(\%)\end{array}$ & $\begin{array}{l}\text { Tree } \\
\text { Size }\end{array}$ \\
\hline $\mathbf{1}$ & $\mathbf{6 . 8 9}$ & $\mathbf{1 0 . 3 4}$ & $\mathbf{2 3}$ & 4.43 & 13.79 & 33 & 3.44 & 13.79 & 35 \\
\hline 2 & 6.89 & 10.34 & 29 & 4.42 & 10.34 & 31 & 3.94 & 13.79 & 35 \\
\hline 3 & 7.88 & 11.49 & 23 & 7.38 & 11.39 & 29 & 5.91 & 12.64 & 31 \\
\hline 4 & 6.89 & 11.49 & 23 & 7.38 & 16.09 & 27 & 5.91 & 17.24 & 29 \\
\hline 5 & 7.88 & 13.79 & 25 & 5.41 & 16.09 & 31 & 3.94 & 14.94 & 33 \\
\hline Average & 7.286 & 11.49 & 24.6 & 5.804 & 13.54 & 30.2 & 4.628 & 14.48 & 32.6 \\
\hline
\end{tabular}

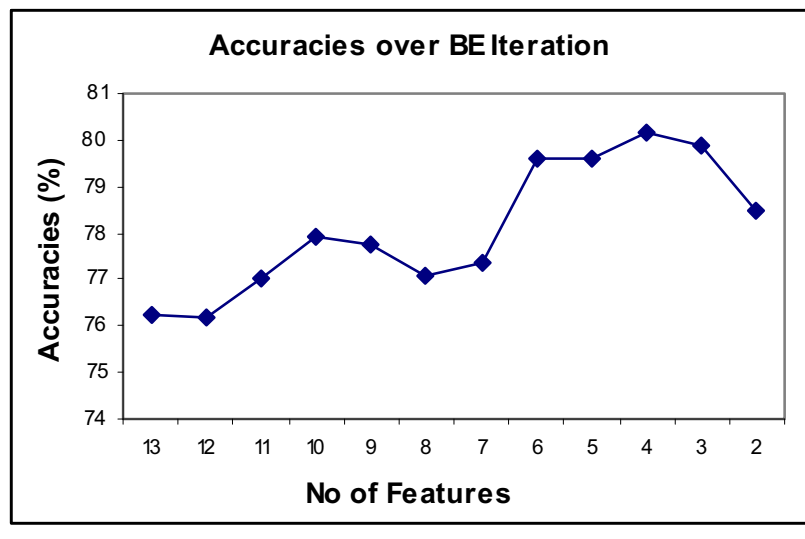

Fig 6. Accuracies over BE iterations in MR-ANNIGMA feature selection.

The data set (Section III) has a total 13 real valued attributes where Ewing 5 tests: HR response to Valsalva manoeuvre (VA_HR), HR variation during deep breathing (DB_HR), BP response to sustained handgrip (HG_BP), immediate HR response to standing (Lying to standing), and BP response to standing (Lying to standing) and ECG features have been included. There are total 291 records and four different classes (1: Atypical, 2: Definite, 3: Early, 4: Normal) in the data. First, the MR-ANNIGMA process has been run for feature selection. The wrapper uses 10 trials to determine the accuracies with 10fold cross validation for each trial in the BE process. The hybrid process (MR-ANNIGMA) starts with 13 attributes and combined score graph has been given in Fig. 4 where feature-1 has the lowest score for ANNIGMA, feature -11 has the lowest MR score and the hybrid finds feature-6 as the lowest (Fig 4). Therefore the hybrid eliminates feature- 6 after the first cycle. In the next cycle, the hybrid re-computes all features' scores resulting in feature-7 attaining the lowest combined score. Therefore it eliminates feature-7 (Fig. 5). The hybrid (MRANNIGMA) continues BE process and achieves the highest accuracy $(80.13 \%)$ for four features (VA_HR, DB_HR, HG_BP and QRS). The detailed accuracies in different iterations of $\mathrm{BE}$ process is given in Fig. 6. Therefore, in the first step, we find four significant features. In the second step, four features with their corresponding data are used to generate decision rules. A SDT $[15,16]$ has been applied on the filtered data. Five separate trials have been performed to generate the final rule set. For each trial, the data has been divided randomly into training set and test set. $30 \%$ of data has been considered for test set and the rest is taken into training in each trial. This has been done for five trials. For each trial, we have computed the decision tree and accuracies for training and test sets. The detail accuracies for five trials have been presented in Table-II. The SDT has also been applied on Ewing's five features and also on all features. It is seen in the Table-II that the proposed approach achieves smallest tree size on average which denotes the most simplified rule sets. While comparing accuracies, it can be seen that the proposed approach achieves the lowest error rate in all test sets. The lowest error rate for test sets and the average smallest tree size denotes the significance of the feature selection algorithm (MRANNIGMA) in the proposed approach. Both the Ewing features set and all features set achieve higher error rate and higher tree size than the proposed approach. Therefore the proposed approach performs better than other approaches and achieves easier decision rules with less number of required tests than Ewing's algorithm. The final decision tree has been chosen for the smallest tree size with the lowest error rate for test set which is found in Trial-1. The decision tree for final rule set is presented in Fig. 7. Rules have been explained in the following.

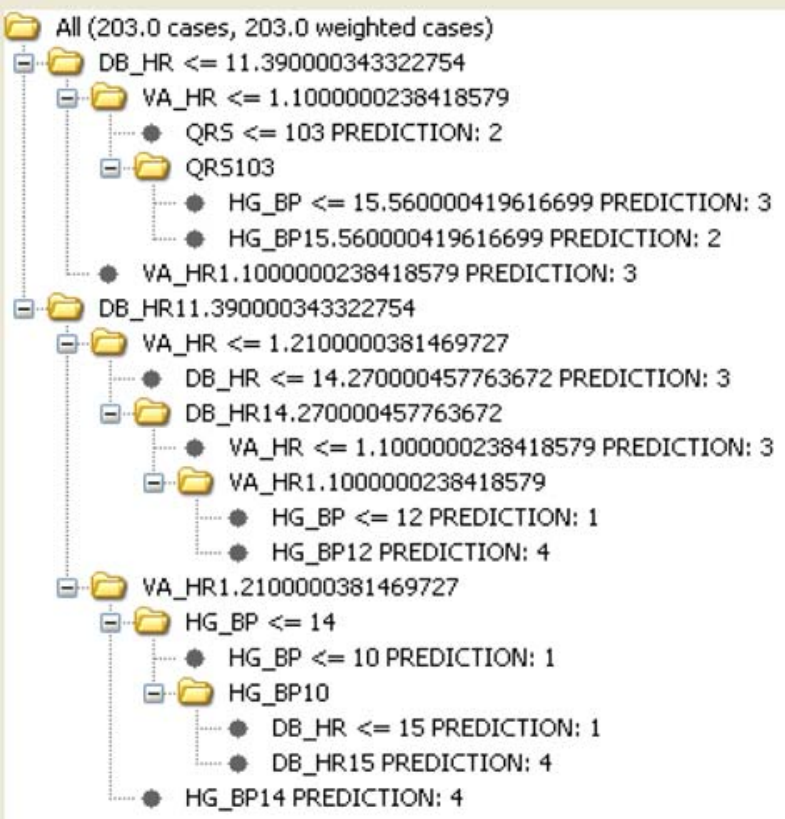

Fig 7. The final decision tree for determination of CAN using four features selected by proposed approach and the SDT algorithm. 
Interpretation of the Rules: The class values are (1=atypical, $2=$ definite, $3=$ Early, $4=$ Normal and $5=$ Severe). The selected features by the proposed approach are DB_HR, VA_HR, QRS, HG_BP. Each path from the root of the decision tree to one of its leaves is transformed to a decision rule by co-joining the intermediate nodes' test-conditions that forms the antecedent part of the rule and class value of the leaf forms the consequent part of the rule. Thus,

The rule for definite CAN is: IF DB_HR $<=11.39$ AND VA_HR $<=1.1$ AND QRS $<=103$ THEN IT IS A 'DEFINITE CAN'.

Or,

IF DB_HR $<=11.39$ AND VA_HR $<=1.1$ AND QRS $>103$ AND HG_BP > 15.56 THEN IT IS A 'DEFINITE CAN'.

The other rules can be extracted from the decision tree in a similar manner.

\section{CONCLUSION}

This paper proposes a novel approach of finding the smallest features set and the simplest decision rule set for identification of Cardiovascular Autonomic Neuropathy (CAN). Standard Ewing's algorithm uses five tests which are difficult to perform clinically and does not use any ECG feature. In this paper, we propose a hybrid of wrapper and filter approaches for finding the most significant features from both Ewing's Features and ECG features of patients' ECG recordings and then apply standard decision tree on those significant features set to generate the rules for determination of CAN. In the proposed approach, a hybrid of filter heuristic (Maximum Relevance, MR) and wrapper heuristic (Artificial Neural Net Input Gain Measurement Approximation ANNIGMA) approach (MR-ANNIGMA) has been used. The novelty of our approaches is that we have involved ECG morphological features in identification of CAN. To the best of our knowledge, the idea of our approach is new and has not been explored yet in the literature. The combined heuristics in the hybrid feature selection (MR-ANNIGMA) take the advantages of the complementary properties of the both filter and wrapper heuristics and guide the wrapper to find optimal and compact feature subsets in the wrapper. The approach has been tested on real clinical records collected in the Charles Sturt University, Australia. Experimental results show that hybrid algorithm (MR-ANNIGMA) finds a smaller feature set than Ewing's algorithm, which is a combination of Ewing's features and ECG features. Application of the obtained feature set by the proposed approach in the rule generation step also achieves less error rate than Ewing's feature set that demonstrates the significance of the proposed approach. Proposed approach would be applied on standard databases of CAN in future.

\section{REFERENCES}

[1] http://www.nlm.nih.gov/medlineplus/ency/article/000776.htm

[2] Ahsan H Khandoker, Herbert F Jelinek and Marimuthu Palaniswami. Identifying diabetic patients with cardiac autonomic neuropathy by heart rate complexity analysis. BioMedical Engineering OnLine 2009; 8:3. Retrieved from http://www.biomedical-engineering-online.com/content/8/1/3

[3] Australian Diabetes Society (ADS) [http://www.diabetessociety.com.au/]

[4] N. Kwak and C. Choi, "Input Feature Selection for Classification Problems", IEEE Transaction on Neural Networks, Vol. 13, No. 1, January 2002

[5] Huda, S, Yearwood, Stranieri, A, "Hybrid wrapper-filter approaches for input feature selection using Maximum relevance and Artificial Neural Network Input Gain Measurement Approximation (ANNIGMA)", The International Conference on Data and Knowledge Engineering (ICDKE),1-3 September 2010, Melbourne, Australia (Accepted)

[6] Ewing DJ, Martyn CN, Young RJ, Clarke BF: The value of cardiovascular autonomic function tests: 10 years experience in diabetes. Diabetes Care 8:491-498, 1985

[7] Ewing, D. J., Campbell, J. W., \& Clarke, B. F. (1980). The natural history of diabetic autonomic neuropathy. Q J Med 49, 95-100.

[8] American Diabetes Association and American Academy of Neurology: Report and recommendations of the San Antonio Conference on diabetic neuropathy (Consensus Statement). Diabetes 37:1000-1004, 1988

[9] Hung-Ta Chen a, Hong-Da Lin b,c, Justin G.S. Wonb,c, Chen-Hsen Lee d,c, Shiao-Chi Wue, Jiun-Dian Lin f, Li-Ying Juan g, Low-Tone Ho d,c, Kam-Tsun Tang d,b,c,," Cardiovascular autonomic neuropathy, autonomic symptoms and diabetic complications in 674 type 2 diabetes, diabetes research and clinical practice 82 (2008)pp 282-290

[10] Peter Stellaa,b, Demetrius Ellisc, Raelene E. Maserd, Trevor J. Orcharda, "Cardiac autonomic neuropathy (expiration and inspiration ratio) in type 1 diabetes Incidence and predictors", Journal of Diabetes and Its Complications 14 (2000) pp 1-6

[11] J.G. Dy and C.E. Brodley, "Feature Subset Selection and Order Identification for Unsupervised Learning," Proc. 17th Int'l Conf. Machine Learning, pp. 247-254, 2000.

[12] C.N. Hsu, H.J. Huang, and D. Schuschel, "The ANNIGMA-Wrapper Approach to Fast Feature Selection for Neural Nets",

[13] A. Jain and D. Zongker, "Feature selection: Evaluation, Application and small sample performance", IEEE Transaction on Pattern Analysis and Machine Intelligence, Vol. 19, No. 2, pp 153-158, Feb 1997.

[14] F. J. Ferri, P. Pudil, M. hatef and J.Kittler, "Comparative study of techniques for large-scale feature selection", Pattern recognition in practice IV, E.S. Gelsema et.al. eds pp 403-413, 1994

[15] J. R. Quinlan, "C4.5 programs for machine learning", Moragn Kaufmann, 1987

[16] J. R. Quinlan, "Simplifying decision trees," Int. J. Man-Mach. Studies, vol. 27, pp. 221-234, 1987. 